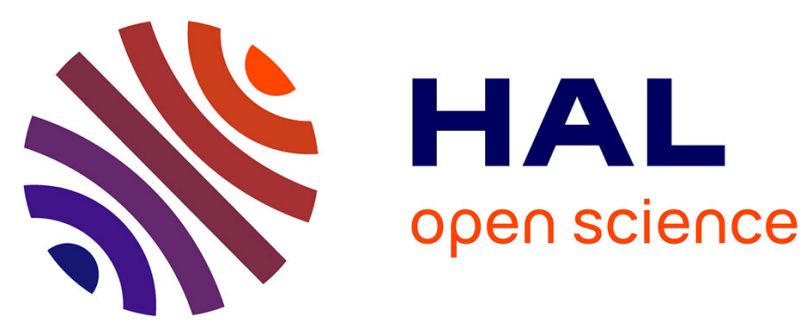

\title{
Spin-Transfer Torque Magnetic Memory as a Stochastic Memristive Synapse for Neuromorphic Systems
}

\author{
Adrien F Vincent, Jérôme Larroque, Nicolas Locatelli, Nesrine Ben \\ Romdhane, Olivier Bichler, Christian Gamrat, Weisheng Zhao, \\ Jacques-Olivier Klein, Sylvie Galdin-Retailleau, Damien Querlioz
}

\section{To cite this version:}

Adrien F Vincent, Jérôme Larroque, Nicolas Locatelli, Nesrine Ben Romdhane, Olivier Bichler, et al.. Spin-Transfer Torque Magnetic Memory as a Stochastic Memristive Synapse for Neuromorphic Systems. IEEE Transactions on Biomedical Circuits and Systems, 2015, 9 (2), pp.166 - 174. 10.1109/TBCAS.2015.2414423 . hal-01822200

\section{HAL Id: hal-01822200 https://hal.science/hal-01822200}

Submitted on 24 Jun 2018

HAL is a multi-disciplinary open access archive for the deposit and dissemination of scientific research documents, whether they are published or not. The documents may come from teaching and research institutions in France or abroad, or from public or private research centers.
L'archive ouverte pluridisciplinaire HAL, est destinée au dépôt et à la diffusion de documents scientifiques de niveau recherche, publiés ou non, émanant des établissements d'enseignement et de recherche français ou étrangers, des laboratoires publics ou privés. 


\title{
Spin-Transfer Torque Magnetic Memory as a Stochastic Memristive Synapse for Neuromorphic Systems
}

\author{
Adrien F. Vincent, Student Member, IEEE, Jérôme Larroque, Nicolas Locatelli, Member, IEEE, \\ Nesrine Ben Romdhane, Olivier Bichler, Christian Gamrat, WeiSheng Zhao, Senior Member, IEEE, \\ Jacques-Olivier Klein, Member, IEEE, Sylvie Galdin-Retailleau, Damien Querlioz, Member, IEEE
}

\begin{abstract}
Spin-transfer torque magnetic memory (STTMRAM) is currently under intense academic and industrial development, since it features nonvolatility, high write and read speed and high endurance. In this work, we show that when used in a non-conventional regime, it can additionally act as a stochastic memristive device, appropriate to implement a "synaptic" function. We introduce basic concepts relating to spin-transfer torque magnetic tunnel junction (STT-MTJ, the STT-MRAM cell) behavior and its possible use to implement learning-capable synapses. Three programming regimes (low, intermediate and high current) are identified and compared. System-level simulations on a task of vehicle counting highlight the potential of the technology for learning systems. Monte Carlo simulations show its robustness to device variations. The simulations also allow comparing the operation of system when the different programming regimes of STT-MTJs are used. With comparison to the high and low current regimes, the intermediate current regime allows minimization of energy consumption, while retaining a high robustness to device variations. These results open the way for unexplored applications of STT-MTJs in robust, low power, cognitive-type systems.
\end{abstract}

Index Terms-modeling, magnetic memories, magnetic devices, simulation.

\section{INTRODUCTION}

Thanks to considerable progress in recent years, spin-transfer torque magnetic random access memory (STT-MRAM) - the second generation of magnetic memory - now appears as a breakthrough for embedded and standalone non-volatile memory, providing fast programming and high endurance [1]. However, a limitation of this technology is its stochastic switching nature [1]-[3]. The time required for programming from a memory state to another is a random quantity, which requires designing programming times with high safety margins, to ensure reliable programming. Device physicists have intensely studied this effect of intrinsic probability [4], [5], and circuit designers have proposed ideas like self-enabled programming to mitigate the issue [2]. However, an alternative approach is

A. F. Vincent, J. Larroque, N. Locatelli, N. Ben Romdhane, W. S Zhao, J.-O. Klein, S. Galdin-Retailleau and D. Querlioz are with Institut d'Electronique Fondamentale, Univ. Paris-Sud, CNRS, 91405 Orsay France (email: damien.querlioz@u-psud.fr).

W.S. Zhao is also with the Spintronics Interdisciplinary Center, Beihang University, Beijing 100191, China.

O. Bichler and C. Gamrat are with CEA, LIST, Saclay, France.

Manuscript received XXX. This work was supported by the ANR COGNISPIN (ANR-13-JS03-0004-01) and the FP7 ICT BAMBI (FP7-ICT-2013-C) projects, and the CNRS/MI DEFI NANO program. to not consider this randomness as a drawback, but as a feature of the device. In particular, here we reinterpret STT-MRAM cell's behavior as a "stochastic memristive device". And we show by system-level simulations how it may be used in a neuromorphic system for practical applications.

In recent years, the exploitation of nanodevices with memory effects (or memristive devices) as synapses in neuromorphic systems has stimulated a growing interest [6]-[21]. They raise the hope for a breakthrough in electronics, bringing smarter, lower power and more adaptive systems. Most of these proposals use memory devices with multi-level capability - the original memristor paradigm [8]. However, an alternative idea is to use binary devices programmed in a stochastic fashion, or even to use binary devices with intrinsic stochastic properties [20], [22]-[24]. In theoretical works, the idea of using stochastic synapses instead of deterministic ones (in a broad sense) has also been proposed with supervised neural networks [25]-[27]. We suggest that STT magnetic memory is ideal for this vision and illustrate it in the case of unsupervised learning.

In the present paper, we introduce the basic physics of STT memory and the foundations of its behavior as a stochastic memristive device. To support the idea, we perform systemlevel simulations incorporating an accurate model of the stochastic effects for an application of car counting. Monte Carlo simulations show the relevance and the robustness of the approach to device variations, and allow us to identify in which regime STT memory should be used.

Partial and preliminary results of this work have appeared in [28]. The present paper adds in-depth discussion on system implications of using STT memory as a synapse, comprehensive Monte Carlo simulations, and compares the operation of the STT-MTJs in different regimes in terms of energy efficiency and robustness to device variations.

\section{Stochastic Memristive Behavior of Magnetic TUNNEL JUNCTIONS}

A magnetic tunnel junction (MTJ, the basic structure of magnetic memory, Figure 1(a)) is composed of a fixed magnetic layer, an oxide layer and a free magnetic layer, whose magnetization can be parallel or antiparallel to the one of the fixed layer. The antiparallel state AP is high resistive and the parallel state $\mathrm{P}$ is low resistive. Thanks to the spin transfer torque (STT) effect, a positive current can switch the STTMTJ from the AP to the $\mathrm{P}$ state, while a negative current can 


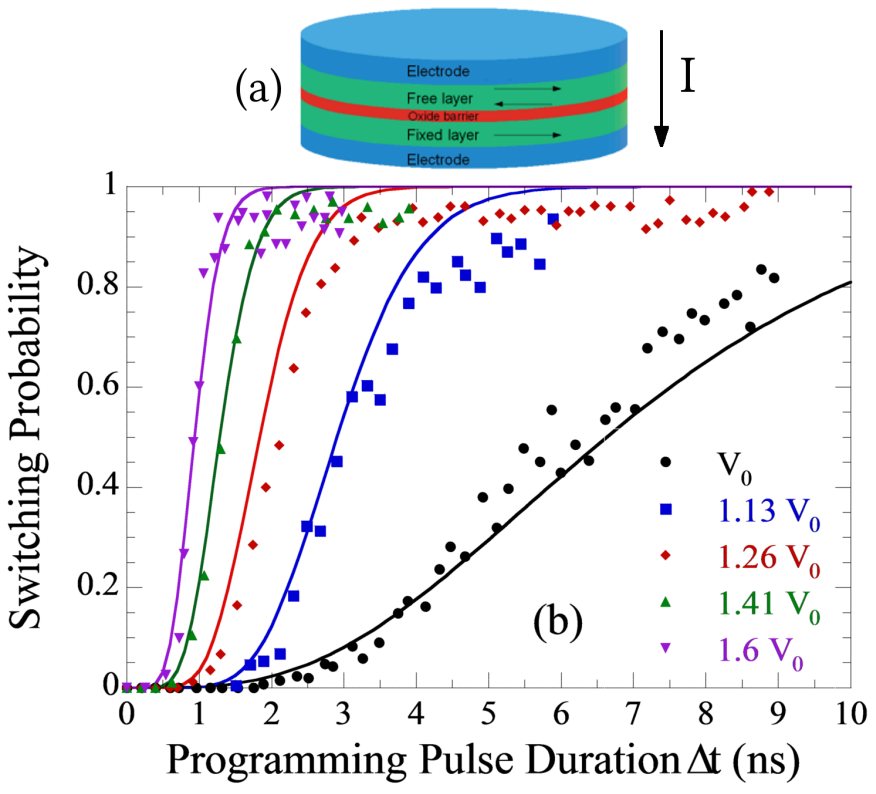

Fig. 1. (a) Schematic illustrating the basic structure of an in-plane magnetic tunnel junction. (b) Symbols: Experimental measurements of probability of switching as a function of programming time, for different programming voltages [5], [29]. Lines: our analytical model.

switch it from the P to the AP state. In this way, a STT-MTJ is extremely reminiscent of a binary bipolar memristor [8].

The switching time depends heavily on the current and is itself a stochastic quantity as observed in the measurements of Figure 1(b). We see that a programming pulse of duration $\Delta t$ has a probability of switching the memory. This stochastic effect is not caused by technological imperfections or filamentary effects like in other resistive memory technologies [22], [24], but is intrinsic to the physics of magnetic switching. This has been largely clarified by magnetism studies.

\section{A. Low Current Regime}

In particular, at low current, memory switching is caused by thermal fluctuations. We can introduce a critical current of a STT-MTJ (in the case of a device with in-plane magnetization):

$$
I_{\mathrm{c}_{0}}=\frac{2|e|}{\hbar} \frac{\alpha V(1 \pm P)}{P} \mu_{0} M_{\mathrm{s}} \frac{M_{\mathrm{eff}}}{2},
$$

where $\alpha, M_{\mathrm{s}}$ and $V$ are the Gilbert damping, the saturation magnetization and the volume of the free layer, $P$ the spin polarization of the current, and $M_{\text {eff }}$ an effective magnetization (all the equations are written in SI units). The sign - in equation 1 is for the $\mathrm{AP} \rightarrow \mathrm{P}$ transition, the sign + for $\mathrm{P} \rightarrow \mathrm{AP}$. It should thus be noted that $I_{\mathrm{c}_{0}}$ has different values for the $\mathrm{P} \rightarrow \mathrm{AP}$ and $\mathrm{AP} \rightarrow \mathrm{P}$ transitions. However, basic calculations confirmed by measurements show that they correspond to the same voltages The symmetry - in voltage - between the $\mathrm{P} \rightarrow \mathrm{AP}$ and $\mathrm{AP} \rightarrow \mathrm{P}$ transitions is an extremely nice property of STT-MTJs used as memristive devices.

If a current $I$ much smaller than $I_{\mathrm{c}_{0}}$ flows through a STTMTJ, the mean switching time $\langle\tau\rangle$ has been proven to behave as the Néel-Brown equation [1]

$$
<\tau>=f_{0}^{-1} \exp \left(\frac{E_{0}}{k_{\mathrm{B}} T}\left(1-\frac{I}{I_{\mathrm{c}_{0}}}\right)\right),
$$

where the energy barrier at zero current is $E_{0}=\mu_{0} M_{\mathrm{s}} H_{\mathrm{k}} V / 2$, $k_{\mathrm{B}} T$ is the thermal energy, $H_{\mathrm{k}}$ the amplitude of anisotropy field and $f_{0}^{-1}$ a constant. Switching time itself is determined by an exponential random law with mean $\langle\tau\rangle$ [1], [4]. This implies that if a programming pulse of duration $\Delta t$ is applied to the junction, its probability of switching is

$$
\mathcal{P}_{\text {sw }}=1-\exp \left(-\frac{\Delta t}{<\tau\rangle}\right) \text {. }
$$

Choosing the pulse duration $\Delta t$ thus allows tuning the switching probability of the devices anywhere between a low $\left(\mathcal{P}_{\text {sw }} \ll 1\right.$ for $\left.\Delta t \ll<\tau>\right)$ and a high probability $\left(\mathcal{P}_{\text {sw }} \approx 1\right.$ for $\Delta t \gg\langle\tau\rangle$ ). It should be noted that STT-MTJ thus possess no hard threshold. Even an extremely low current has a probability to switch the junction, but the mean switching time is exponentially dependent on the current.

\section{B. High Current and Intermediate Current Regimes}

By contrast, when a current much higher than $I_{\mathrm{C}_{0}}$ flows, the physics differs (precessional switching) and the switching time behaves as Sun's law [1], [5]

$$
\tau=\frac{2}{\alpha \gamma \mu_{0} M_{\mathrm{s}}} \frac{I_{\mathrm{c}_{0}}}{I-I_{\mathrm{c}_{0}}} \log \left(\frac{\pi}{2|\theta|}\right),
$$

where $\theta$, the initial angle of the magnetization, is given by a normal random number with mean 0 and standard deviation $\theta_{0}=\sqrt{k_{\mathrm{B}} T /\left(\mu_{0} H_{\mathrm{k}} M_{\mathrm{s}} V\right)}(\gamma$ is the electron gyromagnetic constant). This is no longer an exponential law as is seen for the measurements on Figure 1(b), which are taken in the intermediate and high current regimes.

Although the two regimes $I \ll I_{\mathrm{c}_{0}}$ and $I \gg I_{\mathrm{c}_{0}}$ have been well studied, the intermediate regime is much harder to model. For the present work we developed equations that fit STT-MTJs' behavior in all situations. The relatively complex equations are beyond the scope of the current paper and are published in a sister publication [30]. Figure 1(b) shows that our equations fit experimental measurements.

Figure 2 shows the mean switching time as a function of current (for the $\mathrm{AP} \rightarrow \mathrm{P}$ transition) in all possible regimes, compared with physical simulations based on the macrospin magnetic Landau-Lifschitz-Gilbert-Slonczewski equation with thermal agitation. The low current, high current and intermediate current regimes are visible. This graph also shows how the mean switching time can be tuned over several decades by choosing current or voltage adequately, a unique feature of STT-MTJs.

\section{Integration and Scaling Potential of STT-MTJS}

Although STT-MTJ technology is complex and involves new materials, STT-MTJs are CMOS-compatible, and their integration potential has been demonstrated in several experimental realizations, which use STT-MTJs as standard memory. A standalone memory of $64 \mathrm{Mb}$, in a $90 \mathrm{~nm}$ process, has already reached the market [31], [32], and similar chips have been published in recent years by several groups [33]-[35]. An embedded memory of $1 \mathrm{Mb}$ in a $65 \mathrm{~nm}$ process has been published [36]. 


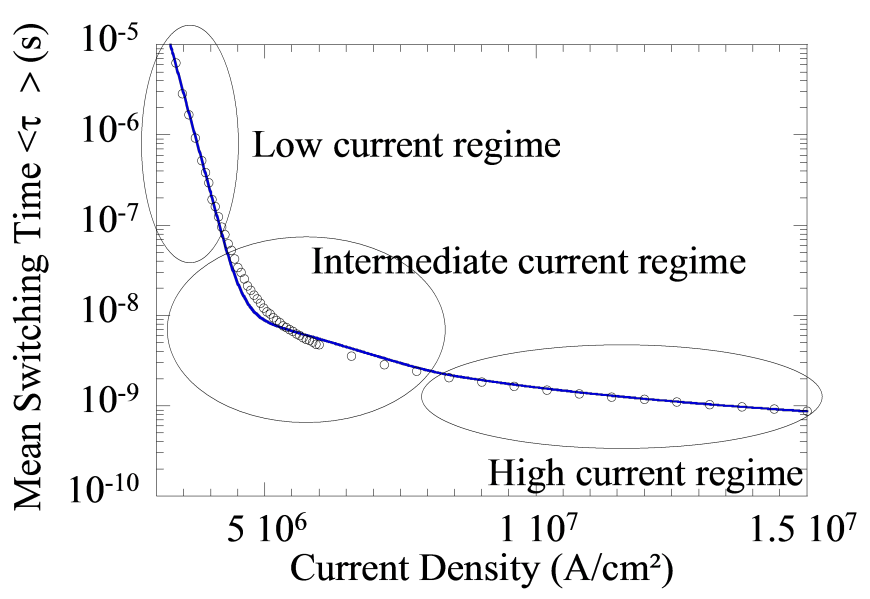

Fig. 2. Mean switching time as a function of current density in a $45 \mathrm{~nm}$ technology STT-MTJ, simulated with our model for the $\mathrm{AP} \rightarrow \mathrm{P}$ transition. Blue line: our analytical model. Symbols: physical simulation based on LandauLifschitz-Gilbert-Slonczewski equation with thermal agitation.

Unlike flash memory, as well as several alternative nonvolatile memory technology, STT-MTJs use programming voltages lesser or equal than logic voltages. Programming currents scale with the technology node [1] and can range from $\mathrm{mA}$ to $10 \mu \mathrm{A}$. The most recent realization use structures where the magnetic layers magnetizations are perpendicular to the structure, which reduces programming current [37]-[39]. In Ref. [36], [37], for example, programming voltage is $0.6 \mathrm{~V}$, programming current is $50 \mu \mathrm{A}$ and programming time only 3 ns. Read and write circuits associated with STT-MTJs have been heavily developed in recent years. Advanced read circuits are sense amplifiers specially designed for STT-MTJs [36], [40]. Advanced write circuits mitigate stochastic effects using self-enabled paradigms [2].

\section{EXAMPLE OF APPLICATION IN A NEUROMORPHiC SYSTEM}

\section{A. Architecture of the System}

In this section, we validate by means of system-level simulation the use of stochastic STT-MTJs as synapses. For this, we adapted a scheme proposed elsewhere for Phase Change Memory [13], [41] and Conductive Bridge RAM (CBRAM) [22], [23]. The system implements a spiking neural network, capable of performing unsupervised learning through a simplified Spike Timing Dependent Plasticity (STDP) rule. Figure 3 shows the basic architecture of the system.

CMOS input neurons present the input as asynchronous spikes, which may come directly from a neuromorphic sensor (e.g. DVS retina [42]). The STT-MTJs are organized as a crossbar connecting input and output neurons. The simplest architecture is to use a passive crossbar (1R), which has also been proposed for memory applications [43]. This scheme is extremely efficient in terms of area, and allows each input neuron to be connected to each output neuron by a single STT-MTJ. Unlike for memory operation, this architecture does not suffer from the sneak path during read operations. Indeed, read operation is naturally done in parallel. (a)

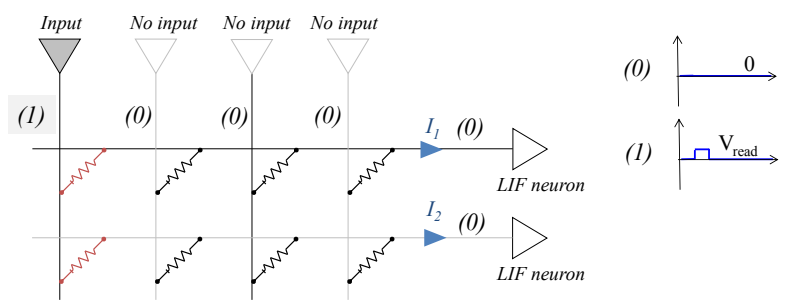

(b)

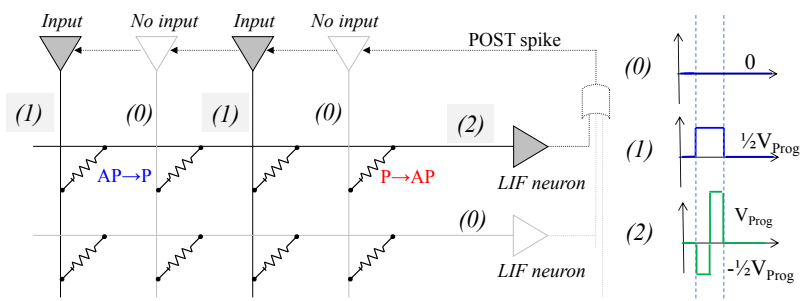

Fig. 3. Schematization of the crossbar architecture (1R case) for the learning system. (a) Read operation, which occurs whenever an input neuron spikes. (b) STDP (write) operation which occurs when an output neuron spikes. Waveforms (1) and (2) are applied concurrently.. Due to the stochastic nature of switching, in the presented example, only two STT-MTJs switch states. .

In contrast, passive crossbar suffers from some sneak paths during programming, which use unnecessary energy, as discussed later. For this reason, this scheme can be adapted to selector devices (1T-1R structure). This loses, unfortunately, the compactness of the scheme. Detailed comparison between $1 \mathrm{R}$ and $1 \mathrm{~T}-1 \mathrm{R}$ will be the subject of future work focused on circuit implementations using STT-MTJs as synapses.

In our system, when an input neuron spikes, it applies a brief read pulse to the crossbar, as illustrated in Figure 3(a). This leads to currents that reach the different output neurons simultaneously. The output neurons, materialized as triangles $(\triangleright)$ in Figure 3 maintain a constant voltage at their input (avoiding sneak path on other STT-MTJs), while reading the current. This can be achieved using second-generation current conveyor designs [57]. The current received by an output neuron depends on the state (P or AP) of the synapse connecting the input to this particular output. The output neurons provide two features. First, using a sense-amplifier type circuit, adapted from the ones used for MRAM [36], [40], the output neuron can determine if an input was received from a P or AP synapse. This process would lead to error if two inputs spiked during a time smaller than the read pulse width. In the practical application that we studied (processing video data from a DVS retina), this situation does not arise. Second, the output neurons integrate the information received from their sense amplifier. Functionally, the output neurons implement leaky integrateand-fire (LIF) spiking neurons. They may be implemented by analog or digital circuits [44]-[48]. Only the inputs coming from $\mathrm{P}$ synapses are integrated by the output neurons. So, MTJs in the AP state act as synapses with a synaptic weight of zero, and MTJs in the P state act as synapses with a synaptic weight of one.

When an output neuron spikes, it inhibits the other output neurons: their internal variable is reset to zero. In practice, this can be implemented by nearest-neighbor schemes like diffusor 
network [49]. With this lateral inhibition, our architecture is reminiscent of a winner-takes-all neural network.

\section{B. Simplified STDP Rule}

Additionally, when an output neuron spikes it applies a voltage pulse on the crossbar, that implements our learning rule inspired by STDP. STDP is a model for synaptic learning in the brain [50], [51]. Many works have proposed to use memory devices to implement STDP [9], [11]-[14], [52]-[57], usually involving sophisticated schemes. Here we use an extremely simplified version adapted to stochastic STT-MTJs.

This simplified STDP rule deviates from biological models, and includes two significant simplifications. First, STDP occurs only when an output neuron spikes, which differs from most STDP models, but is easier to implement with nanodevices as seen in [12], [13], [23], [58]. In the field of neuroscience, a similar choice has been taken by Nessler et al. [59]. They showed that in this condition, STDP can approximate the powerful machine learning algorithm of Expectation-Maximization. Second, our STDP rule is stochastic instead of deterministic: a STDP event has a probability to switch a nanodevice.

In practice, STDP steps act as the programming operations of the network and are presented in Figure 3(b). When an output neuron spikes, the whole system enters a "programming" phase. The output neuron that spiked applies the voltage waveform (2) to its row of the crossbar, while the inputs that were active in a recent time window apply an input pulse (1). The combination of these voltage pulses implements a simplified STDP rule: when an output neuron spikes, a STT-MTJ connected to it

- has a given probability of switching to the $\mathrm{P}$ state if its input neuron was active in a recent time window;

- has a given probability of switching to the AP state if its input neuron was not active in the same time window.

At the same time, the STT-MTJs connected to the other output neurons are either non-selected, or half-selected (i.e. the voltage applied to these devices is either 0 or $V_{\text {Prog }} / 2$ ). According to the model of section II, the switching probability of these devices is negligible.

This probabilistic rule is similar to the one that we proposed for CBRAM [22], [23]. However, the voltage waveforms are simpler because of the symmetry in voltage between $\mathrm{P} \rightarrow \mathrm{AP}$ and $\mathrm{AP} \rightarrow \mathrm{P}$ switching in MTJs. The learning rule is also reminiscent of the simplified STDP proposed for computational neuroscience studies in [59], but in a binary and probabilistic version. As shown further, our simplified STDP allows a form of powerful unsupervised learning.

\section{Methodology for System-Level Simulation}

To validate the use of STT-MTJs as synapses, we performed system-level simulations where STT-MTJs are modeled accurately according to the model of section II. We used a specialized neuromorphic spiking neural network simulator developed in our lab. The simulation is time-step based and simulates CMOS circuits functionally. The simulator is designed for the possibility of including comprehensive physical models for the nanodevices and for their imperfections. This

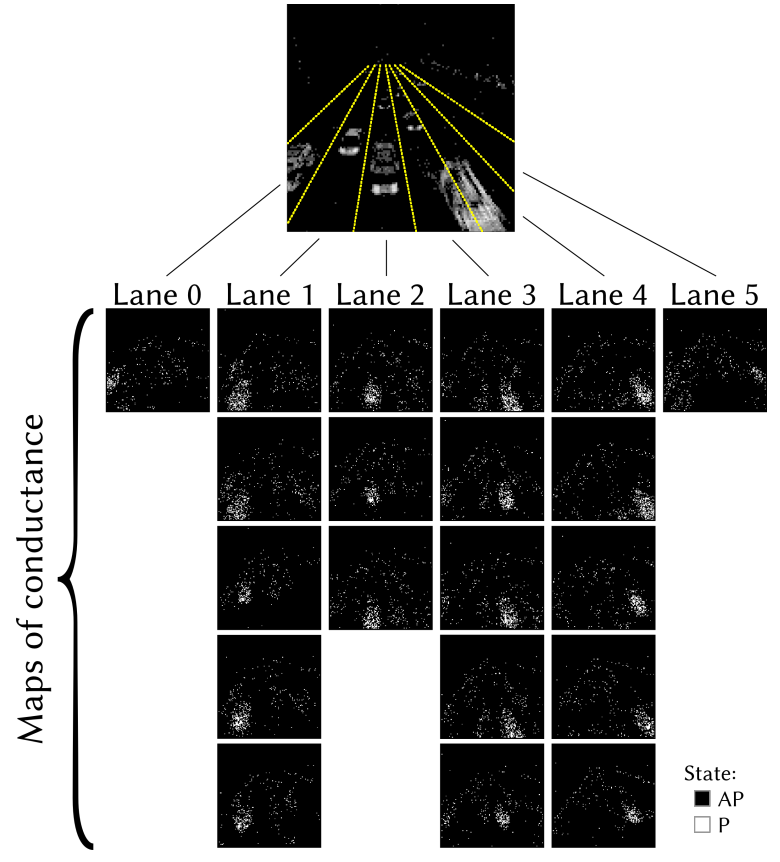

Fig. 4. Representation of the final state of the MTJs. Every subimage represents one output neuron. The state of the MTJs is organized as the input pixels in the image. White is P, black is AP state.

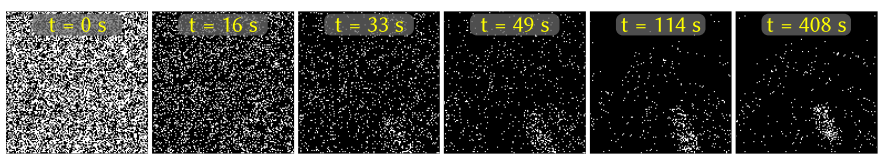

Fig. 5. Evolution of the states of the MTJs connected to a one of the output neurons during the learning process, and plotted as in Figure 4.

kind of simulation is much faster than SPICE simulation; for our test application, the simulation time was between 25 and 40 times the real time on a Xeon E5620 processor. This allows us to simulate practical applications, and to perform statistical studies (Monte Carlo simulations).

We based our simulations on a STT-MTJ device representative of a $45 \mathrm{~nm}$ technology. The STT-MTJs are ellipses with a width of $40 \mathrm{~nm}$, a length of $100 \mathrm{~nm}$ and a free layer thickness of $2 \mathrm{~nm}$. The tunnel magnetoresistance is $150 \%$ (i.e. $\left.R_{\mathrm{AP}} / R_{\mathrm{P}}=2.5\right)$.

The programming voltage $V_{\text {Prog }}$ in Figure $3(\mathrm{~b})$ is varied between $0.3 \mathrm{~V}$ and $0.6 \mathrm{~V}$, in order to study its impact of the system. Under the constraint of fixed switching probability, the programming pulses duration heavily depends on their amplitude $V_{\text {Prog. }}$. Thus, with the presented geometry and a switching probability of $10 \%$, it ranges from $32.3 \mu$ s (with $V_{\text {Prog }}=0.3 \mathrm{~V}$ ) to $3.9 \mathrm{~ns}$ (with $V_{\text {Prog }}=0.6 \mathrm{~V}$ ). Concerning the reading pulses, the are $1 \mathrm{~ns}$ long and $0.1 \mathrm{~V}$ high, resulting in an insignificant probability of parasitic switching.

\section{Task Results for Car Detection}

For our test application, we presented an 80 seconds video of cars passing on a freeway recorded with a neuromorphic retina [42] (the video is freely available online [60]). This retina, directly inspired by the human retina, works asynchronously 
and without frames, and generates spikes when the intensity of a pixel changes. Each pixel of the retina comes in two versions, sensitive to increase and decrease of pixel itensity, respectively.

Each input neuron of our system corresponds to one of the 32,768 pixels of the retina. The system has 20 output neurons and each of them is connected to every input pixel through a synapse made of a single STT-MTJ. We presented the video five times and observed that due to the simplified STDP learning rule, the output neurons naturally specialize on particular lanes and the system effectively becomes a vehicle counter.

The specialization of the output neurons is evident in Figure 4, which shows the final states of the STT-MTJs. The top image represents sample input: we plotted as white every input that spiked during a $30 \mathrm{~ms}$ period. The yellow dotted lines materialize the different lanes of the freeway. Every other image represents the final states of the STT-MTJs connected to one of the output neurons (white is P, black is AP). The output neurons are listed according to the lane to which they specialized. The number of output neurons specialized in a given lane is not uniform (lanes 1, 3 and 5 have five specialized output neurons, lane 2 three output neurons, and lanes 0 and 5 only one). This is determined by the number of cars passing on each lane, and the number of pixels activated by a car passing on each lane. In particular, fewer vehicles are passing on lanes 0 and 5 than on other lanes.

Figure 5 shows how a pattern emerges and stabilizes starting from uniform random distribution of synaptic states, in one of the output neurons. Originally, the STT-MTJ states are random. After $33 \mathrm{~s}$, the neuron has started to specialize in lane 4. After $114 \mathrm{~s}$, the states of the STT-MTJs are stabilized.

Once the learning has been achieved, we can deactivate both lateral inhibition and the learning steps, causing the operation of the system to become very low power. Disabling lateral inhibition and learning is not inspired by biology, but motivated by engineering purpose. Disabling lateral inhibition slightly increases the detection rate of the system, allowing the system to react to two vehicles passing at the exact same time. Disabling learning decreases the power consumption of the system. Once the system has learnt, it can also be switched OFF and ON without losing its function, since the state of STT-MTJs is nonvolatile.

To estimate the performance of the system on the car detection task, for each line, we chose the output neuron with the best detection rate. This operation can be done automatically by adding a second layer to the network as proposed in [61].

We first consider a situation where the devices are programmed in the intermediate current regime with a voltage $V_{\text {Prog }}=0.46 \mathrm{~V}$, and where device variation is neglected. The STDP programming pulse width is adjusted such that transitions from AP to $\mathrm{P}$ and $\mathrm{P}$ to the AP both have a probability of $10 \%$.

If we interpret the system as a vehicle counter, the detection rate is $97.3 \%$ for the four inward lanes. Detection rate is $62 \%$ and $28 \%$ for the two outward lanes. The proportion of false positives among the output spikes is $4.7 \%$.

If we do not stop learning and inhibition once the system is stable, the detection rate for the four inward lanes is sightly reduced to $94.6 \%$, while the proportion of false positives is

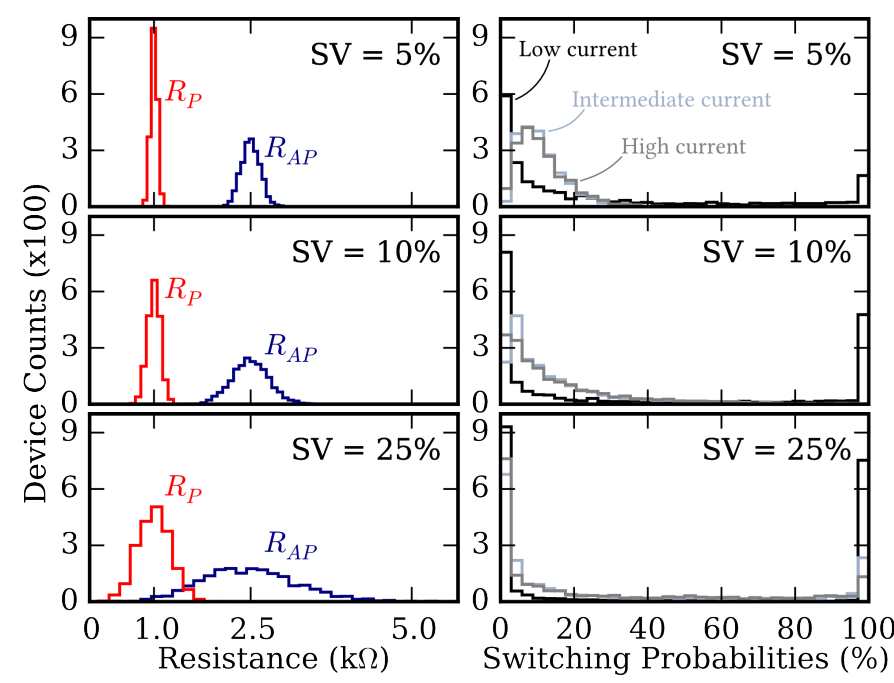

Fig. 6. Histograms representing the values of $\mathrm{P}$ and $\mathrm{AP}$ states resistance (left subfigures) and switching probabilities (right subfigures) when synaptic variability is introduced. The switching probabilities are represented in the low, intermediate and high programming current regimes (obtained with $V_{\text {Prog }}=$ $0.3 \mathrm{~V}, 0.4 \mathrm{~V}$ and $0.6 \mathrm{~V}$ respectively). Switching probability is $10 \%$ in all cases for $\mathrm{SV}=0$. From top to bottom, synaptic variability SV is $5 \%, 10 \%$ and $25 \%$ of relative standard deviation (one-sigma) on resistance of the $\mathrm{P}$ state and TMR.

$5.0 \%$.

The best result on the same dataset, using a neural network with double precision analog weight reports a detection rate of $98.1 \%$ and a proportion of false positives of $4.3 \%$ [61].

During the learning process, the power consumption for programming the STT-MTJs (excluding the power consumption of the CMOS neurons and of the rest of the system) is only $180 \mathrm{nW}$, therefore making low power operation possible. We show further that this figure depends tremendously on the chosen programming regime.

Sneak paths represent only a small fraction of the power consumption $(8.4 \%)$. This is caused by the high parallelism of the STDP operation. However, the proportion of sneak path energy increases linearly with the number of output neurons. The overall power consumption is smaller than in the case of CBRAMs used for solving a similar task [23] due to the low voltage operation and high speed of STT-MTJs in the probabilistic regime.

Interestingly, the performance of the system is only weakly dependent on the actual switching probability of STT-MTJs during learning. If we adjust the programming pulse so that $\mathrm{P}$ to AP transitions have a probability of 5\% (P to AP remaining $10 \%$ ), the system detection rate for the four inward lanes drops to $86 \%$.

\section{Impact of Device Properties on the NEUROMORPHIC SYSTEM}

\section{A. Impact of Device Variations}

In reality, device variations cause different STT-MTJs to have different probabilities of switching when being applied the same programming pulses. To evaluate the robustness of our approach to this issue, we performed Monte Carlo simulations. 
Variations of the minimum and maximum resistances of the MTJs are considered. Since they affect the current that flow through the devices, this kind of variation has a dramatic effect on the probabilities of switching STT-MTJs when programming pulses are applied. This is illustrated in Figure 6. We introduce several levels of Gaussian variability on the resistance of the $\mathrm{P}$ state and on the TMR of 2000 STT-MTJs. The synaptic variability SV parameters, $5 \%, 10 \%$ or $25 \%$, represent the relative standard deviation $(\sigma / \mu$, one-sigma) of resistance of the $\mathrm{P}$ state and TMR. This way of introducing variability is motivated by experimental realizations, which suggest that variations on the resistance of the $\mathrm{P}$ state and on the TMR are uncorrelated and have equivalent relative standard deviation [33], [34]. In Refs. [33], [34], the SV parameter was found to be approximately $5 \%$. The degree of variability that we are considering therefore corresponds to extremely high level of variability, in terms of realistic technology. The left subfigures of Figure 6 represent histograms on the values of the resistances of the parallel and antiparallel states. The right subfigures are computed with the model of section II and represent histograms of the switching probabilities of STT-MTJs, for programming pulses whose probability to program STT-MTJs when $\mathrm{SV}=0$ is $10 \%$. Three histograms are superimposed in the low, intermediate and high current regimes. We see that the variability on the switching probabilities is exacerbated with regards to the variability on the resistance states. The variability on the switching probabilities is also considerably higher in the low programming current regime than in the intermediate and high current regimes.

When simulating the whole system, we observed spectacular tolerance of the system to device variation. We first consider the case where the STT-MTJs are programmed in the intermediate current regime. In Figure 7(a), we plotted detection rate and proportion of false positives as a function of synaptic variability in two situations: a situation where we did not stop STDP at the end of learning, and a situation where we stopped it. Without variability, the situation where learning is not stopped has a significantly reduced detection rate compared to when it is disabled. Up to $17 \%$ of of synaptic variability, no impact is seen on the detection rate in both situations, while as seen in Figure 6, the variability of switching probability is considerable. The degree of robustness is astonishing. When synaptic plasticity is increased above $17 \%$, the detection rate drops sharply in the cases where learning and inhibition are disabled, and more gently in the case where they are not. The case where inhibition is not disabled is more robust, because in the presence of high device variation, some output neurons react preferentially. Inhibition limits their activity.

Figure 7(b) shows the proportion of false positives in the same conditions as Figure 7(a). Similar trends are seen, although the number of false positives starts to increase at a lower synaptic variability (13\%) than the detection rate.

Finally, we have also considered transient variations of the properties of the STT-MTJs. We performed simulations where, every time a STT-MTJ is programmed, its device properties $\left(R_{P}\right.$ and TMR) are redrawn randomly with a synaptic variability of $10 \%$. This constitutes an artificially high degree of variations since MTJs properties are experimentally stable from
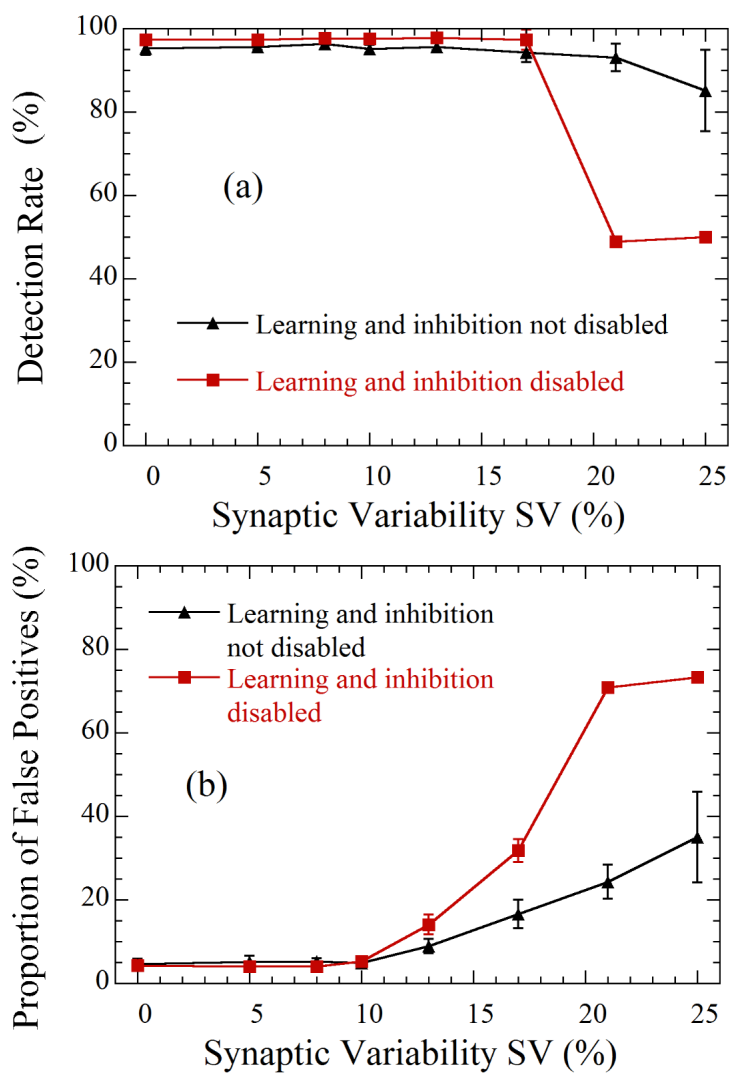

Fig. 7. Detection rate (a) and proportion of false positives (b) as a function of synaptic variability in a situation where learning is disabled at the end of the learning process (squares) or not (triangles). Every simulation was repeated ten times, the error bars represent one standard deviation.

cycle to cycle. Nevertheless, in these conditions our system achieved the same detection rate and number of false positives as in the situation were the MTJs properties are stable.

\section{B. Impact of the Programming Regime}

We have seen in section II that STT-MTJs can be operated in different regimes (low, intermediate and high current). In this part, system level simulations allow us to understand the benefits and drawbacks of the different regimes when using STT-MTJs as synapses.

Without device variations, all regimes allow implementing the same switching probability, and thus lead to the same detection rate and proportion of false positives. However, the energy required to program the STT-MTJs differs significantly. In the intermediate current regime, with a programming voltage of $0.46 \mathrm{~V}$, programming power is $180 \mathrm{nW}$. In the high current regime, with a programming voltage of $0.6 \mathrm{~V}$, it reaches $220 \mathrm{nW}$. In the low current regime, with a programming voltage of $0.3 \mathrm{~V}$, it reaches a higher $0.42 \mathrm{~mW}$.

In the presence of device variation, the programming regimes are nonequivalent. This is seen in the Monte Carlo simulations of Figure 8. In the intermediate and high current regimes, the detection rate is robust until a synaptic variability of $17 \%$; it is robust until only $10 \%$ in the low current regimes. The number of false positives is robust up to $10 \%$ of device variability in 

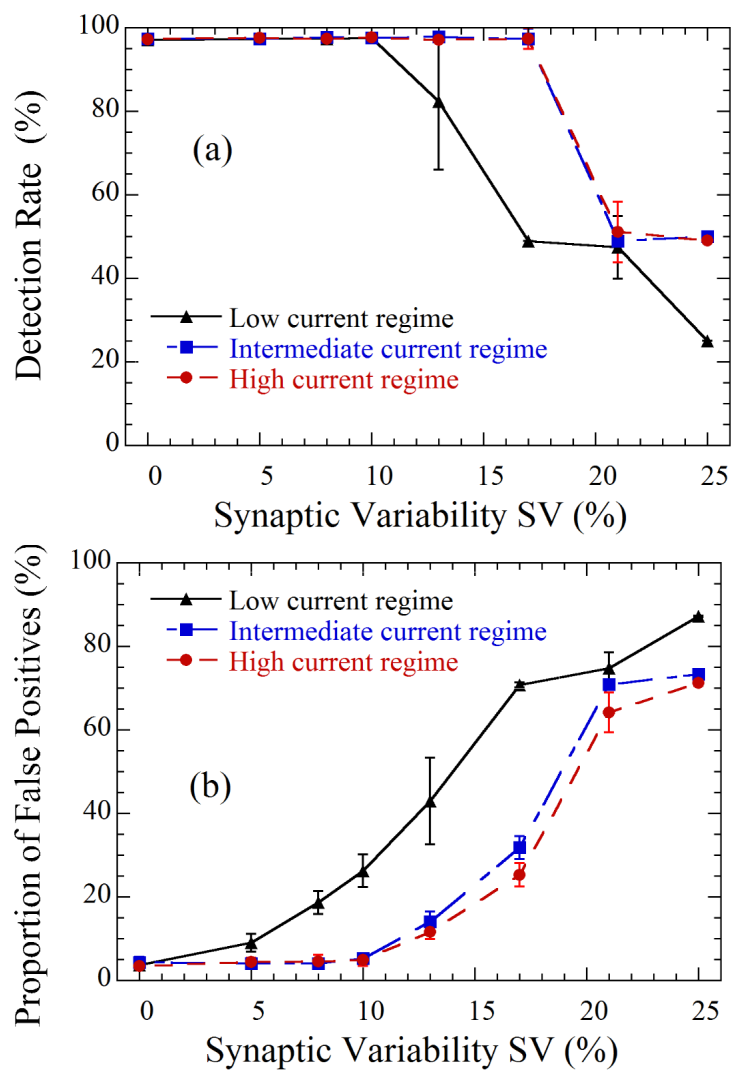

Fig. 8. Detection rate (a) and proportion of false positives (b) as a function of synaptic variability with STT-MTJs programmed in the low (triangles), intermediate (squares) or high (circles) programming regimes. Learning was disabled at the end of the learning process. Every simulation was repeated ten times, the error bars represent one standard deviation.

the cases of intermediate and high programming current, while it increases as soon as there are device variations in the low programming current.

This contrast can be explained by the device physics of STTMTJs. As seen in section II, in the low current regime, the mean switching time of STT-MTJs has an exponential dependence on the current. In the presence of device variations, each STTMTJ is programmed with a different current, and therefore has an extremely disparate switching probability. For intermediate and high programming currents, the mean switching time of STT-MTJs has a softer dependence on current than in the low programming current regime.

In summary, intermediate and high programming currents require much smaller programming power than the low programming current and have a better robustness to device variations. Since the intermediate programming current uses smaller voltages than the high programming current regime, STT-MTJs have a better reliability in this regime. The intermediate programming therefore appears as the ideal regime for synaptic use of STT-MTJs. This differs from traditional memory applications of STT-MTJs, where programming speed is the major concern and where the high programming current may be a better choice.

\section{CONCLUSION}

In this work, we interpreted magnetic tunnel junctions' behavior as a stochastic memristive synapse. The stochastic effects were modeled accurately using analytical physical equations. The mean switching time can be tuned over many decades, and STT-MTJs can be programmed in three different regimes. Low voltages are used for programming and are symmetric between positive and negative polarizations. We introduced a neural network-inspired system that can exploit this stochastic effect to perform unsupervised learning. The switching probabilities of the nanodevices do not need to be controlled perfectly, as the system is robust to device mismatch, which is evidenced by Monte Carlo simulations. The intermediate programming current regime minimizes energy consumption and leads to high robustness to device variations. This regime appears to be ideal for the use of STT-MTJs as synapses.

Future works address the optimized circuits for using STTMTJs as synapses, which might differ from the circuits used for using STT-MTJs as memory [62], and the physical realization of hybrid CMOS/stochastic synapse circuits. From a system point of view, the architecture should be advanced for demonstrating more complex applications.

This work also gives insight into a new way to use memristive nanodevices. Unpredictability caused by nanoscale physics is not necessarily an enemy but can become the foundation for efficient processing using novel computing paradigms.

\section{ACKNOWLEDGEMENTS}

The authors would like to thank T. Devolder, D. Ravelosona, P. Bessiere, J. Droulez, A. Mizrahi, D. Vodenicarevic, J. S. Friedman and J. Grollier for fruitful discussions.

\section{REFERENCES}

[1] Z. Diao, Z. Li, S. Wang, Y. Ding, A. Panchula, E. Chen, L.-C. Wang, and Y. Huai, "Spin-transfer torque switching in magnetic tunnel junctions and spin-transfer torque random access memory," Journal of Physics: Condensed Matter, vol. 19, no. 16, p. 165209, Apr. 2007.

[2] Y. Lakys, W. S. Zhao, T. Devolder, Y. Zhang, J.-O. Klein, D. Ravelosona, and C. Chappert, "Self-Enabled "Error-Free" Switching Circuit for Spin Transfer Torque MRAM and Logic," IEEE Trans. Magn., vol. 48, no. 9, pp. 2403-2406, 2012.

[3] Y. Zhang, W. Zhao, G. Prenat, T. Devolder, J.-O. Klein, C. Chappert, B. Dieny, and D. Ravelosona, "Electrical Modeling of Stochastic Spin Transfer Torque Writing in Magnetic Tunnel Junctions for Memory and Logic Applications," IEEE Transactions on Magnetics, vol. 49, no. 7, pp. 4375-4378, Jul. 2013.

[4] D. Bedau, H. Liu, J. Z. Sun, J. A. Katine, E. E. Fullerton, S. Mangin, and A. D. Kent, "Spin-transfer pulse switching: From the dynamic to the thermally activated regime," Applied Physics Letters, vol. 97, no. 26, pp. 262 502-262 502-3, Dec. 2010.

[5] T. Devolder, J. Hayakawa, K. Ito, H. Takahashi, S. Ikeda, P. Crozat, N. Zerounian, J.-V. Kim, C. Chappert, and H. Ohno, "Single-Shot TimeResolved Measurements of Nanosecond-Scale Spin-Transfer Induced Switching: Stochastic Versus Deterministic Aspects," Phys. Rev. Lett. vol. 100, no. 5, p. 057206, Feb. 2008.

[6] Y. V. Pershin, S. La Fontaine, and M. Di Ventra, "Memristive model of amoeba learning," Phys. Rev. E, vol. 80, no. 2, p. 021926, 2009.

[7] S. H. Jo, K.-H. Kim, and W. Lu, "Programmable Resistance Switching in Nanoscale Two-Terminal Devices," Nano Lett., vol. 9, no. 1, pp. 496-500, Jan. 2009

[8] D. B. Strukov, G. S. Snider, D. R. Stewart, and R. S. Williams, "The missing memristor found," Nature, vol. 453, no. 7191, pp. 80-83, May 2008. 
[9] G. S. Snider, "Self-organized computation with unreliable, memristive nanodevices," Nanotechnol., vol. 18, no. 36, p. 365202, Sep. 2007.

[10] S. Bamford, A. Murray, and D. Willshaw, "Spike-Timing-Dependent Plasticity With Weight Dependence Evoked From Physical Constraints," IEEE Transactions on Biomedical Circuits and Systems, vol. 6, no. 4, pp. 385-398, Aug. 2012.

[11] K. Seo, I. Kim, S. Jung, M. Jo, S. Park, J. Park, J. Shin, K. P. Biju, J. Kong, K. Lee, B. Lee, and H. Hwang, "Analog memory and spiketiming-dependent plasticity characteristics of a nanoscale titanium oxide bilayer resistive switching device," Nanotechnol., vol. 22, no. 25, p. 254023, Jun. 2011.

[12] D. Querlioz, O. Bichler, and C. Gamrat, "Simulation of a memristorbased spiking neural network immune to device variations," Proc. of the Int. Joint Conf. on Neural Networks (IJCNN), pp. 1775 - 1781, 2011.

[13] M. Suri, O. Bichler, D. Querlioz, O. Cueto, L. Perniola, V. Sousa, D. Vuillaume, C. Gamrat, and B. DeSalvo, "Phase change memory as synapse for ultra-dense neuromorphic systems: Application to complex visual pattern extraction,” in IEDM Tech. Dig. IEEE, Dec. 2011, pp. 4.4.1-4.4.4.

[14] S. Yu, Y. Wu, R. Jeyasingh, D. Kuzum, and H. P. Wong, "An Electronic Synapse Device Based on Metal Oxide Resistive Switching Memory for Neuromorphic Computation," IEEE Trans. Electron Dev., vol. 58, no. 8 , pp. 2729-2737, Aug. 2011.

[15] V. Erokhin, T. Berzina, P. Camorani, A. Smerieri, D. Vavoulis, J. Feng, and M. P. Fontana, "Material Memristive Device Circuits with Synaptic Plasticity: Learning and Memory," BioNanoScience, vol. 1, no. 1-2, pp. 24-30, Apr. 2011

[16] A. Chanthbouala, V. Garcia, R. O. Cherifi, K. Bouzehouane, S. Fusil, X. Moya, S. Xavier, H. Yamada, C. Deranlot, N. D. Mathur, M. Bibes, A. Barthélémy, and J. Grollier, "A ferroelectric memristor," Nat. Mat., vol. 11 , no. 10 , pp. $860-864,2012$

[17] G. Indiveri, B. Linares-Barranco, R. Legenstein, G. Deligeorgis, and T. Prodromakis, "Integration of nanoscale memristor synapses in neuromorphic computing architectures," Nanotechnology, vol. 24, no. 38, p. 384010, Sep. 2013.

[18] M. Sharad, C. Augustine, G. Panagopoulos, and K. Roy, "Spin-Based Neuron Model With Domain-Wall Magnets as Synapse," IEEE Transactions on Nanotechnology, vol. 11, no. 4, pp. 843 -853, Jul. 2012

[19] D. Chabi, D. Querlioz, W. Zhao, and J.-O. Klein, "Robust Learning Approach for Neuro-inspired Nanoscale Crossbar Architecture," J. Emerg. Technol. Comput. Syst., vol. 10, no. 1, pp. 5:1-5:20, Jan. 2014.

[20] O. Kavehei, "Highly Scalable Neuromorphic Hardware with 1-bit Stochastic nano-Synapses," arXiv e-print 1309.6419, Sep. 2013.

[21] S. Saïghi, C. G. Mayr, T. Serrano-Gotarredona, H. Schmidt, G. Lecerf, J. Tomas, J. Grollier, S. Boyn, A. F. Vincent, D. Querlioz, S. La Barbera, F. Alibart, D. Vuillaume, O. Bichler, C. Gamrat, and B. Linares-Barranco, "Plasticity in memristive devices for spiking neural networks," Front. Neurosci., vol. 9, p. 51, 2015.

[22] M. Suri, O. Bichler, D. Querlioz, G. Palma, E. Vianello, D. Vuillaume, C. Gamrat, and B. DeSalvo, "CBRAM Devices as Binary Synapses for Low-Power Stochastic Neuromorphic Systems: Auditory (Cochlea) and Visual (Retina) Cognitive Processing Applications," IEDM Tech. Dig., p. 10.3.1, 2012.

[23] M. Suri, D. Querlioz, O. Bichler, G. Palma, E. Vianello, D. Vuillaume, C. Gamrat, and B. DeSalvo, "Bio-Inspired Stochastic Computing Using Binary CBRAM Synapses," IEEE Transactions on Electron Devices, vol. 60, no. 7, pp. 2402-2409, 2013

[24] S. Gaba, P. Sheridan, J. Zhou, S. Choi, and W. Lu, "Stochastic memristive devices for computing and neuromorphic applications," Nanoscale, vol. 5, no. 13, pp. 5872-5878, Jun. 2013.

[25] J. H. Lee and K. K. Likharev, "Defect-tolerant nanoelectronic pattern classifiers," Int. J. Circuit Theory Appl., vol. 35, no. 3, pp. 239-264, May 2007

[26] W. Senn and S. Fusi, "Convergence of stochastic learning in perceptrons with binary synapses," Physical Review E, vol. 71, no. 6, p. 061907, Jun. 2005.

[27] Y. Kondo and Y. Sawada, "Functional abilities of a stochastic logic neural network," IEEE Transactions on Neural Networks, vol. 3, no. 3 pp. $434-443$, May 1992.

[28] A. F. Vincent, J. Larroque, W. S. Zhao, N. Ben Romdhane, O. Bichler, C. Gamrat, J.-O. Klein, S. Galdin-Retailleau, and D. Querlioz, "Spintransfer torque magnetic memory as a stochastic memristive synapse," in 2014 IEEE International Symposium on Circuits and Systems (ISCAS), Jun. 2014, pp. 1074-1077.

[29] M. Marins de Castro, R. C. Sousa, S. Bandiera, C. Ducruet, A. Chavent, S. Auffret, C. Papusoi, I. L. Prejbeanu, C. Portemont, L. Vila, U. Ebels, B. Rodmacq, and B. Dieny, "Precessional spin-transfer switching in a magnetic tunnel junction with a synthetic antiferromagnetic perpendicular polarizer," J. Appl. Phys., vol. 111, no. 7, pp. 07C912-07C912-3, Mar 2012.

[30] A. F. Vincent, N. Locatelli, J.-O. Klein, W. S. Zhao, S. Galdin-Retailleau, and D. Querlioz, "Analytical Macrospin Modeling of the Stochastic Switching Time of Spin-Transfer Torque Devices," IEEE Transactions on Electron Devices, vol. 62, no. 1, pp. 164-170, Jan. 2015.

[31] N. Rizzo, D. Houssameddine, J. Janesky, R. Whig, F. Mancoff, M. Schneider, M. DeHerrera, J. Sun, K. Nagel, S. Deshpande et al., "A fully functional $64 \mathrm{mb}$ ddr3 st-mram built on $90 \mathrm{~nm}$ cmos technology," Magnetics, IEEE Transactions on, vol. 49, no. 7, pp. 4441-4446, 2013.

[32] T. Andre, S. Alam, D. Gogl, C. Subramanian, H. Lin, W. Meadows, X. Zhang, N. Rizzo, J. Janesky, D. Houssameddine, and J. Slaughter, "Stmram fundamentals, challenges, and applications," in Custom Integrated Circuits Conference (CICC), 2013 IEEE, Sept 2013, pp. 1-8.

[33] R. Beach, T. Min, C. Horng, Q. Chen, P. Sherman, S. Le, S. Young, K. Yang, H. Yu, X. Lu, W. Kula, T. Zhong, R. Xiao, A. Zhong, G. Liu, J. Kan, J. Yuan, J. Chen, R. Tong, J. Chien, T. Torng, D. Tang, P. Wang, M. Chen, S. Assefa, M. Qazi, J. DeBrosse, M. Gaidis, S. Kanakasabapathy, Y. Lu, J. Nowak, E. O'Sullivan, T. Maffitt, J. Sun, and W. Gallagher, "A statistical study of magnetic tunnel junctions for high-density spin torque transfer-MRAM (STT-MRAM)," in Electron Devices Meeting, 2008. IEDM 2008. IEEE International, Dec. 2008, pp. $1-4$.

[34] D. Worledge, G. Hu, P. Trouilloud, D. Abraham, S. Brown, M. Gaidis, J. Nowak, E. O'Sullivan, R. Robertazzi, J. Sun et al., "Switching distributions and write reliability of perpendicular spin torque mram," in Electron Devices Meeting (IEDM), 2010 IEEE International. IEEE, 2010, pp. 12-5.

[35] S. Chung, K.-M. Rho, S.-D. Kim, H.-J. Suh, D.-J. Kim, H. Kim, S. Lee, J.-H. Park, H.-M. Hwang, S.-M. Hwang et al., "Fully integrated $54 \mathrm{~nm}$ stt-ram with the smallest bit cell dimension for high density memory application," in Electron Devices Meeting (IEDM), 2010 IEEE International. IEEE, 2010, pp. 12-7.

[36] H. Noguchi, K. Kushida, K. Ikegami, K. Abe, E. Kitagawa, S. Kashiwada, C. Kamata, A. Kawasumi, H. Hara, and S. Fujita, "A 250-mhz 256b-i/o 1 -mb stt-mram with advanced perpendicular mtj based dual cell for nonvolatile magnetic caches to reduce active power of processors," in VLSI Technology (VLSIT), 2013 Symposium on, June 2013, pp. C108_ C109.

[37] E. Kitagawa, S. Fujita, K. Nomura, H. Noguchi, K. Abe, K. Ikegami, T. Daibou, Y. Kato, C. Kamata, S. Kashiwada, N. Shimomura, J. Ito, and H. Yoda, "Impact of ultra low power and fast write operation of advanced perpendicular mtj on power reduction for high-performance mobile cpu," in Electron Devices Meeting (IEDM), 2012 IEEE International, Dec 2012, pp. 29.4.1-29.4.4.

[38] J. H. Kim, W. Lim, U. Pi, J. Lee, W. Kim, J. Kim, K. Kim, Y. Park, S. Park, M. Kang, Y. Kim, W. Kim, S. Kim, J. Park, S. Lee, Y. Lee, J. Yoon, S. Oh, S. Park, S. Jeong, S. Nam, H. Kang, and E. Jung, "Verification on the extreme scalability of stt-mram without loss of thermal stability below $15 \mathrm{~nm}$ mtj cell," in VLSI Technology (VLSI-Technology): Digest of Technical Papers, 2014 Symposium on, June 2014, pp. 1-2.

[39] L. Thomas, G. Jan, J. Zhu, H. Liu, Y.-J. Lee, S. Le, R.-Y. Tong, K. Pi, Y.-J. Wang, D. Shen, R. He, J. Haq, J. Teng, V. Lam, K. Huang, T. Zhong, T. Torng, and P.-K. Wang, "Perpendicular spin transfer torque magnetic random access memories with high spin torque efficiency and thermal stability for embedded applications (invited)," Journal of Applied Physics, vol. 115 , no. 17 , pp.,- 2014.

[40] W. Zhao, C. Chappert, V. Javerliac, and J.-P. Noziere, "High speed, high stability and low power sensing amplifier for mtj/cmos hybrid logic circuits," Magnetics, IEEE Transactions on, vol. 45, no. 10, pp. 3784-3787, Oct 2009.

[41] O. Bichler, M. Suri, D. Querlioz, D. Vuillaume, B. DeSalvo, and C. Gamrat, "Visual Pattern Extraction Using Energy-Efficient "2-PCM Synapse" Neuromorphic Architecture," IEEE Trans. Electron Devices, vol. 59, no. 8, pp. 2206 - 2214, 2012.

[42] P. Lichtsteiner, C. Posch, and T. Delbruck, "A 128x 128120 dB 15 mus Latency Asynchronous Temporal Contrast Vision Sensor," IEEE J. Solid-State Circuits, vol. 43, no. 2, pp. 566-576, 2008.

[43] W. Zhao, S. Chaudhuri, C. Accoto, J.-O. Klein, C. Chappert, and P. Mazoyer, "Cross-Point Architecture for Spin-Transfer Torque Magnetic Random Access Memory," IEEE Transactions on Nanotechnology, vol. 11, no. 5, pp. 907 -917, Sep. 2012.

[44] G. Indiveri, B. Linares-Barranco, T. J. Hamilton, R. Etienne-Cummings, T. Delbruck, S.-C. Liu, P. Häfliger, S. Renaud, J. Schemmel, G. Cauwenberghs, J. Arthur, S. Saighi, J. Wijekoon, and K. Boahen, "Neuromorphic 
silicon neuron circuits," Front. Neuromorphic Engineering, vol. 5, p. 73 2011.

[45] P. A. Merolla, J. V. Arthur, R. Alvarez-Icaza, A. S. Cassidy, J. Sawada, F. Akopyan, B. L. Jackson, N. Imam, C. Guo, Y. Nakamura, B. Brezzo, I. Vo, S. K. Esser, R. Appuswamy, B. Taba, A. Amir, M. D. Flickner, W. P. Risk, R. Manohar, and D. S. Modha, "A million spiking-neuron integrated circuit with a scalable communication network and interface," Science, vol. 345, no. 6197, pp. 668-673, Aug. 2014.

[46] S. Saighi, Y. Bornat, J. Tomas, G. Le Masson, and S. Renaud, "A Library of Analog Operators Based on the Hodgkin-Huxley Formalism for the Design of Tunable, Real-Time, Silicon Neurons," IEEE Transactions on Biomedical Circuits and Systems, vol. 5, no. 1, pp. 3-19, Feb. 2011.

[47] S. Mitra, S. Fusi, and G. Indiveri, "Real-Time Classification of Complex Patterns Using Spike-Based Learning in Neuromorphic VLSI," IEEE Transactions on Biomedical Circuits and Systems, vol. 3, no. 1, pp. 32-42, 2009.

[48] Y. Wang and S.-C. Liu, "Active Processing of Spatio-Temporal Input Patterns in Silicon Dendrites," IEEE Transactions on Biomedical Circuits and Systems, vol. 7, no. 3, pp. 307-318, Jun. 2013.

[49] J. V. Arthur and K. A. Boahen, "Learning in silicon: Timing is everything," Advances in neural information processing systems, vol. 18, pp. 281-1185, 2006.

[50] H. Markram, J. Lubke, M. Frotscher, and B. Sakmann, "Regulation of Synaptic Efficacy by Coincidence of Postsynaptic APs and EPSPs," Science, vol. 275, no. 5297, pp. 213-215, Jan. 1997.

[51] G.-Q. Bi and M.-M. Poo, "Synaptic modification by correlated activity: Hebb's Postulate Revisited," Аnпи. Rev. Neurosci., vol. 24, no. 1, pp. 139-166, Mar. 2001.

[52] G. Snider, "Spike-timing-dependent learning in memristive nanodevices," in Prof. of IEEE International Symposium on Nanoscale Architectures 2008 (NANOARCH), 2008, pp. 85-92.

[53] B. Linares-Barranco and T. Serrano-Gotarredona, "Exploiting memristance in adaptive asynchronous spiking neuromorphic nanotechnology systems," in Proc. of IEEE Conference on Nanotechnology, 2009, 2009, pp. 601-604.

[54] S. H. Jo, T. Chang, I. Ebong, B. B. Bhadviya, P. Mazumder, and W. Lu, "Nanoscale Memristor Device as Synapse in Neuromorphic Systems," Nano Lett., vol. 10, no. 4, pp. 1297-1301, Apr. 2010.

[55] F. Alibart, S. Pleutin, O. Bichler, C. Gamrat, T. Serrano-Gotarredona, B. Linares-Barranco, and D. Vuillaume, "A Memristive Nanoparticle/Organic Hybrid Synapstor for Neuroinspired Computing," Advanced Functional Materials, vol. 22, no. 3, pp. 609-616, 2012.

[56] A. Afifi, A. Ayatollahi, and F. Raissi, "Implementation of biologically plausible spiking neural network models on the memristor crossbar-based CMOS/nano circuits," in European Conference on Circuit Theory and Design (ECCTD), 2009, pp. 563-566.

[57] G. Lecerf, J. Tomas, and S. Saighi, "Excitatory and Inhibitory Memristive Synapses for Spiking Neural Networks," in 2013 IEEE International Symposium on Circuits and Systems (ISCAS), 2013, pp. 1616-1619.

[58] D. Querlioz, O. Bichler, P. Dollfus, and C. Gamrat, "Immunity to Device Variations in a Spiking Neural Network with Memristive Nanodevices," IEEE Trans. Nanotechnol., vol. 12, no. 3, pp. 288 - 295, 2013.

[59] B. Nessler, M. Pfeiffer, L. Buesing, and W. Maass, "Bayesian Computation Emerges in Generic Cortical Microcircuits through Spike-TimingDependent Plasticity," PLoS Computational Biology, vol. 9, no. 4, Apr. 2013.

[60] http://sourceforge.net/p/jaer/wiki/AER data/.

[61] O. Bichler, D. Querlioz, S. J. Thorpe, J.-P. Bourgoin, and C. Gamrat, "Extraction of temporally correlated features from dynamic vision sensors with spike-timing-dependent plasticity," Neural Networks, vol. 32, pp. 339-348, 2012.

[62] Y. Zhang, W. Zhao, J.-O. Klein, W. Kang, D. Querlioz, Y. Zhang, D. Ravelosona, and C. Chappert, "Spintronics for low-power computing," in Design, Automation and Test in Europe Conference and Exhibition (DATE), 2014, Mar. 2014, pp. 1-6.

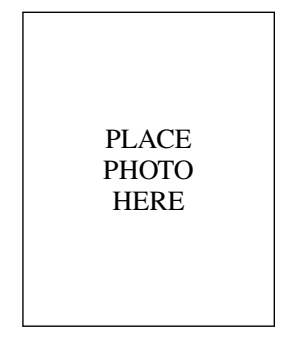

Adrien F. Vincent received the M.S. degree from the École Normale Supérieure de Cachan, France, in 2013. During his Ph.D. at Univ. Paris-Sud, he is studying the integration of spintronic nanodevices in neuromorphic architectures.

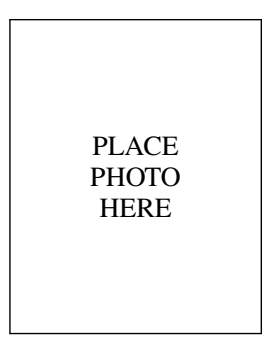

Jérôme Larroque received the M.S. degree from Univ. Paris-Sud, France, in 2012. He worked on the use of magnetic tunnel junctions as synapses. During his Ph.D. at Univ. Paris-Sud, he is studying nanometer-scaled thermoelectric generators.

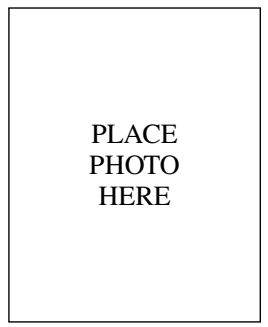

Nicolas Locatelli received the $\mathrm{Ph} . \mathrm{D}$. degree from the University of Paris-Sud, Orsay, France, in 2012, with a focus on developing conventional or more innovative bioinspired applications of spintronic nanodevices. He studied the dynamics of coupled magnetic vortices nanooscillators.
PLACE

PHOTO

HERE

PLACE

PHOTO

HERE
Nesrine Ben Romdhane received the Electronic Engineering degree from Engineers National School of Tunisia in 2007. From 2007 to 2011, she worked in STMicroelectronics of Tunis as a Non Volatile Memories Designer Engineer. She is currently working at Univ. Paris-Sud, France in a research project on emerging non-volatile memories integration with logic blocks, in embedded processor for healthcare and smart grids applications.

Olivier Bichler received the M.S. degree in embedded systems from the École Normale Superieure de Cachan, France, in 2009 and the Ph. D. degree from the Universite Paris-Sud, Orsay, France, in 2012. He is now a Research Engineer at CEA LIST, France, and develops novel architectures based on nanoelectronics and bio-inspired neuromorphic computing. 


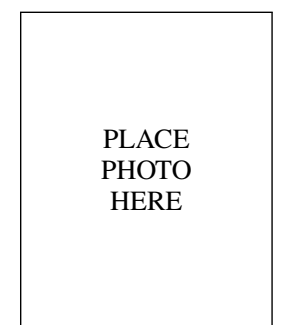

Christian Gamrat received a degree in electrical engineering from the Université Joseph Fourier, Grenoble, France, in 1979 and a degree in information processing in 1993 from École Nationale Supérieure dâĂŹElectronique et de Radioélectricité, Grenoble, France. In 1981, he started his career at CEA/DSM Grenoble on the design of high speed data acquisition systems for solid state and nuclear physics experiments, got involved in the study and design of neural networks computing machines in 1987, and led the team for the MIND-1024 neurocomputer project in 1989. In 1994, he finalized the development of the SYMPHONIE embedded massively parallel computer for use on board military fighter aircraft. In 1997 he started activity on hardware reconfigurable computing, and in 2003, he initiated research on novel computing architectures aimed at nanotechnologies. $\mathrm{He}$ is currently a Senior Expert in the field of advanced computing architectures and nanocomputing, and he leads the Nanocomputing group with CEA LIST, Gif-sur-Yvette, France.

\begin{tabular}{|c|}
\hline \\
\\
PLACE \\
PHOTO \\
HERE \\
\end{tabular}

Weisheng Zhao received the Ph.D. degree in physics from the Univ. Paris-Sud, France, in 2007. Before joining the CNRS in 2009 as a tenured research scientist, he investigated spintronic devices based logic circuits. He currently works on the hybrid integration of nanodevices with CMOS circuit and new non-volatile memory, and architecture design. $\mathrm{He}$ has authored or co-authored more than 80 scientific papers.

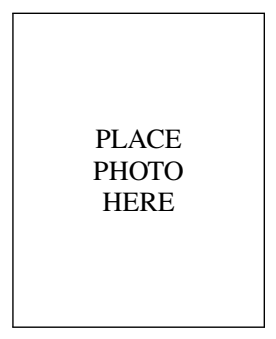

Jacques-Olivier Klein received the Ph.D. degree from the Univ. Paris-Sud, France, in 1995. He is currently Professor at Univ. Paris-Sud, where he leads the nano-computing research group focusing on the architecture of circuits and systems based on emerging nanodevices in the field of nanomagnetism and bio-inspired nanoelectronics. He is author of 70 technical papers including 7 invited communications.

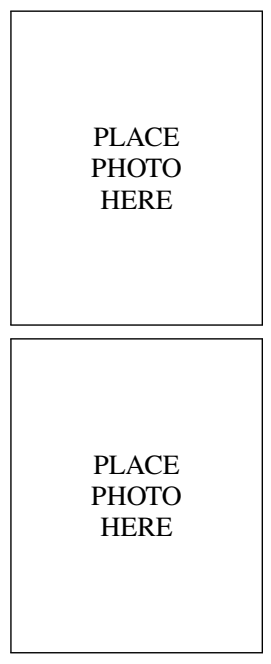

Sylvie Galdin-Retailleau received the Ph.D. degree from the Univ. Paris-Sud, France, in 1992. Since 2001, she has been a Professor at Univ. ParisSud. Since 2011, she has been the Dean of the Faculty of Sciences, Univ. Paris-Sud. She worked on Monte-Carlo simulations and quantum effects in electronic nanodevices. Her current interest is on novel applications of magnetic nanodevices.

Damien Querlioz received the M. S. degree from École Normale Supérieure, Paris in 2005 and the $\mathrm{Ph}$.D. degree from the Univ. Paris-Sud, France, in 2008. After postdocs at Stanford University and at CEA LIST, he became a CNRS research scientist with Univ. Paris-Sud in 2010. He develops new concepts in nanoelectronics and spintronics relying on bioinspiration. He leads the ANR CogniSpin project, which investigates the use of magnetic memory as synapses. He leads the CNRS/MI DEFIBAYES project and is a one of the lead PI of the FP7 FETOPEN BAMBI project, which explore the new paradigms for nanolectronics based on Bayesian inference. 